Acta Crystallographica Section A

Foundations of Crystallography

ISSN 0108-7673

Received 2 December 2011

Accepted 18 January 2012

(C) 2012 International Union of Crystallography Printed in Singapore - all rights reserved

\section{Enumeration of one-dimensional crystal structures obtained from a minimum of diffraction intensities}

\author{
Ahmed Al-Asadi, ${ }^{a, b}$ Eugene Chudin $^{c}$ and Oleg V. Tsodikov ${ }^{a *}$

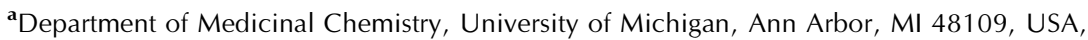

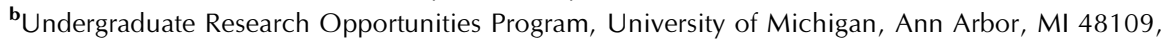 \\ USA, and ${ }^{\mathbf{c}} 12719$ Via Neive, San Diego, CA 92130, USA. Correspondence e-mail: \\ olegt@umich.edu
}

\begin{abstract}
A central problem in crystallography is crystal structure determination directly from diffraction intensities. For structures of small molecules, this problem has been addressed by probabilistic direct methods that allow one to obtain the structure coordinates with a high degree of certainty given a sufficiently large set of intensities. In contrast, deterministic algebraic methods that could guarantee a solution and may be applicable to macromolecules have not yet emerged. In this study a basic algebraic question is posed: how many crystal structures can be obtained from a given set of intensities? Recently, by using a new origin definition and the method of elementary symmetrical polynomials, all small $(N$ $\leq 4$ atoms) one-dimensional crystal structures that could be obtained from the minimum set of $N-1$ lowest-resolution intensities were enumerated. Here, by using methods of modern algebraic geometry the maximum number of onedimensional crystal structures that can be determined from the minimum set of intensities for $N>4$ is obtained. It is demonstrated that this ambiguity increases exponentially with the increasing number of atoms in the structure $N\left(\sim 4^{N} / N^{3 / 2}\right.$ for $N>>1$ ) and includes non-homometric structures. Therefore, a minimum set of intensities, even in principle, is insufficient for structure determination for all but very small structures.
\end{abstract}

\section{Introduction}

Determining a crystal structure from a single set of diffraction intensities without an anomalous signal or a similar structure is a central problem in crystallography with applications to other imaging technologies. It has been known since the pioneering studies by Ott (1927) and Avrami (1938) that an atomic model of a crystal structure can, in principle, be obtained solely from diffraction intensities when the number of such intensities does not exceed greatly the number of unknown coordinates of the atoms in the structure. Algebraic methodologies of determining crystal structures from intensities have been undergoing further development (Cervellino \& Ciccariello, 1996, 1999, 2001, 2005; Pilz \& Fischer, 1998, 1999, 2000; Fischer \& Pilz, 1997); however a practical algebraic method of crystal structure determination has not yet emerged. Importantly, general ideas from Ott's and Avrami's studies spurred development of direct methods, starting from the determination of structures of centrosymmetric crystals by using HarkerKasper inequalities (Harker \& Kasper, 1947, 1948; Gillis, $1948 a, b)$ and culminating in the powerful methodology developed by Karle and Hauptman in the 1950s (Hauptman \& Karle, 1957; Karle \& Hauptman, 1957). Today, software based on these direct methods (most notably, SHELX and $S n B$ ) is used for determining structures of most small molecules and is gaining popularity in determining substructures of heavy atoms and anomalous scatterers in macromolecular crystallography (Sheldrick, 2008; Miller et al., 1994; Rappleye et al., 2002; Weeks \& Miller, 1999).

Because of their probabilistic character, direct methods do not absolutely guarantee that a structure can be obtained. The likelihood of determining structures larger than a thousand or so atoms by these methods is vanishingly small, precluding their use in macromolecular structure determination with the exception of ultra high resolution $(<1.2 \AA)$ structures of very small proteins. It is clear that an approach that is distinct from the traditional direct methodology is required for obtaining larger structures solely from intensities. The rapid improvement in computer technology and progress in modern computational mathematics led to re-examination of previously intractable complex problems in many areas of technology. In particular, it is time to re-evaluate the possibility of development of a deterministic approach of obtaining a crystal structure from a single set of intensities.

The first question that needs to be answered in assessing such methods is a long-standing one: how many structures can be determined from a given set of intensities? It is known that even the idealized complete set of perfectly measured intensities cannot yield a unique structure due to enantiomeric and homometric ambiguities (Patterson, 1939, 1944). It was 
recently rigorously proven that at most two homometric onedimensional crystal structures of $N=4$ equal atoms can be obtained (Shkel et al., 2011). Only lower bounds on the number of such structures are known for $N=5$ and $N=6$ (Bullough, 1963) and no information about crystal structure ambiguity is available, to our knowledge, for larger $N$ or for any $N$ in the cases of two- and three-dimensional crystals of equal atoms, whereas an example of three-dimensional homometric structures has been known for 80 years (Pauling \& Shappell, 1930). The number of crystal structures that yield (or can be obtained from) a realistic, incomplete, set of intensities is larger, because it may include non-homometric structures. Recently, we investigated the ambiguity of structure determination for small $(N \leq 4)$ one-dimensional crystal structures of equal atoms given the minimum set of lowestresolution intensities, by applying the method of elementary symmetric polynomials with a new origin definition (Shkel et al., 2011). The ambiguity has not been investigated for larger $N$. Here we report the analysis of the number of onedimensional crystal structures of any number of equal atoms that can be obtained from the minimum of diffraction intensity data, by using approaches of modern computational algebraic geometry.

\section{Enumeration of one-dimensional crystal structures obtained from a minimum of intensities}

\subsection{Theoretical background}

Normalized structure factors $F_{h}$ describing X-ray diffraction from a one-dimensional crystal of $N$ equal point atoms located at fractional coordinates $x_{j}\left(0 \leq x_{j}<1, j=1,2, \ldots, N\right)$ of the unit cell are

$F_{h}=\sum_{j=1}^{N} \exp \left(2 \pi i h x_{j}\right)=\sum_{j=1}^{N} \xi_{j}^{h}, h=-\infty, \ldots,-1,0,1, \ldots,+\infty$,

where $\xi_{j}=\exp \left(2 \pi i x_{j}\right)$ is a natural periodic coordinate.

In the experiment, only intensities $I_{h}$ are measured, which are proportional to the square of the amplitude of $F_{h}$. Therefore, after appropriate normalization, these intensities are given by the following system of equations:

$$
I_{h}=\left|F_{h}\right|^{2}=\left(\sum_{j=1}^{N} \xi_{j}^{h}\right)\left(\sum_{j=1}^{N} \xi_{j}^{-h}\right) \quad h=0,1, \ldots,+\infty .
$$

Owing to the periodicity, the intensity values are invariant with respect to the location of the coordinate origin. Once the origin location is specified, $N-1$ coordinates become independent unknown variables in system (2) taken together with the equation defining the origin. Therefore, structure determination will require a minimum of $N-1$ intensity values. The choice of the origin has a profound effect on the functional form of system (2), which will become clear in this study. We will use the two following origin definitions to enumerate structures that can be obtained from solving system (2) for the minimum lowest-resolution set of intensities $I_{h}, h=1, \ldots$,
$N-1$ : (i) the origin coincides with the center of mass of the structure (Shkel et al., 2011) and (ii) the origin coincides with one of the atoms.

\subsection{Crystal structure ambiguity analysis for the origin at the center of mass of the structure}

In a previous study from our group, the one-dimensional crystal structure determination problem was formulated for the origin located at the center of mass of the structure (Shkel et al., 2011). This formalism, summarized as Appendix $A$ in the supplementary material, ${ }^{\mathbf{1}}$ allows one to reduce this structure determination problem to a system of polynomial equations (A5) in Appendix A. The number of solutions of system (A5) was previously analyzed by standard elimination techniques of elementary algebra for $N \leq 4$ (Shkel et al., 2011). We demonstrated that system (A5) has two, six and 16 solutions for $N=2,3$ and 4, respectively. For $N>4$, both the elementary methods and advanced Grobner basis techniques [as implemented in the powerful software $\operatorname{CoCoA}$ (CoCoA team; http:// cocoa.dima.unige.it) and Mathematica (Wolfram Research)] could not simplify this system due to its steeply increasing complexity with increasing $N$. Therefore, we used methods of modern computational algebraic geometry to count the number of solutions of system (A5). Specifically, for each equation of the system, we constructed a geometrical object called a Newton polytope and applied Bernstein's theorem to this collection of Newton polytopes. The basics of this methodology are summarized in Appendix $B$ in the supplementary material. We will geometrically illustrate this method for $N=2$ and $N=3$.

For $N=2$, system (A5) contains only one equation: $I_{1}=e_{1}^{2}$, or $e_{1}^{2}-I_{1}=0$ in the standard form. The Newton polytope for the polynomial on the left-hand side is a line segment from 0 to 2 . The mixed volume in this case is simply the volume of this polytope, or the length of the segment, equal to 2. Indeed, there are two solutions in this case. For $N=3$, system (A5) consists of two equations:

$$
\begin{aligned}
& e_{1} e_{2}-I_{1}=0 \\
& e_{1}^{2} e_{2}^{2}-2\left(e_{1}^{3}+e_{2}^{3}\right)+4 e_{1} e_{2}-I_{2}=0 .
\end{aligned}
$$

This problem is two dimensional as there are two variables, $e_{1}$ and $e_{2}$; therefore one needs to calculate the mixed area for this system. The Newton polytopes for the second polynomial and for the Minkowski sum of the two polynomials of this system are shown in Fig. 1. The area of the first two-point polytope is zero; therefore the mixed area, which is equal to the number of roots of this system, is the difference between the area of the Minkowski sum polytope and that of the second polytope, i.e. $12-6=6$ (Fig. 1), in agreement with the previous result (Shkel et al., 2011). Because the dimensionality of system (A5), $N-1$, increases steeply with increasing structure size, the calculations of the Newton polytopes (the convexity operation on a number of points) and their volumes are

\footnotetext{
${ }^{1}$ Appendices $A, B$ and $C$ are available from the IUCr electronic archives (Reference: SC5048). Services for accessing these data are described at the back of the journal.
} 
Table 1

Mixed volumes and the number of unique one-dimensional crystal structures that can be obtained from the minimum of intensities.

\begin{tabular}{lllll}
\hline & $\begin{array}{l}V_{\text {mix }}, \\
\text { system (A5) }\end{array}$ & $\begin{array}{l}n_{\text {uniq }}, \\
\text { system (A5) } \dagger\end{array}$ & $\begin{array}{l}V_{\text {mix }}, \\
\text { system (4) }\end{array}$ & $\begin{array}{l}n_{\text {uniq }}, \\
\text { system (4) } \ddagger\end{array}$ \\
\hline 2 & 2 & $1 \S$ & 2 & 1 ฯ \\
3 & 6 & 1 & 12 & 1 \\
4 & 16 & 2 & 120 & 2.5 \\
5 & 60 & 6 & 1680 & 7 \\
6 & 180 & 15 & 30240 & 21 \\
7 & 714 & 51 & 665280 & 66 \\
8 & 2432 & 152 & 17297280 & 214.5 \\
9 & & & 518918400 & 715 \\
10 & & & 17643225600 & 2431 \\
11 & & & $6.70443 \times 10^{11}$ & 8398 \\
12 & & & $2.81586 \times 10^{13}$ & 29393 \\
\hline
\end{tabular}

$\dagger$ The number of unique solutions of system (A5), $n_{\text {uniq }}=V_{\text {mix }} /(2 N)$. $\ddagger$ The approximate number of unique solutions of system (4), $n_{\text {uniq }}=V_{\text {mix }} /(2 N$ !). In this column, fractional numbers arise as a result of this division, because $V_{\text {mix }}$ is an approximation of the number of solutions of system (4). $\S$ For $N=2$ and the origin in the center of mass, the enantiomer of a structure coincides with the structure; hence here $n_{\text {uniq }}=V_{\text {mix }} / 2$. For $N=2$ and the origin in one of the atoms, the enantiomer of a structure is equivalent to choosing the origin in the other atom; hence here $n_{\text {uniq }}=$ $V_{\text {mix }} / 2$

difficult problems in modern algebra. We used the robust Quickhull algorithm (Barber et al., 1996) as implemented in the software $Q H U L L$ (http://www.qhull.org) to calculate mixed volumes for $N \leq 8$; for larger $N$, these calculations were prohibitively time consuming. These mixed volumes are given in Table 1. Even though, in general, the mixed volume is a tight upper bound on the number of solutions, if the coefficients in front of the monomial terms are generic, the mixed volume is equal to the number of solutions of a polynomial system. Because the mixed volumes given in Table 1 coincide with the previously calculated number of solutions for $N \leq 4$ (as given above; Shkel et al., 2011), the monomial coefficients in system (A5) for any $N$ are likely generic. The generic form of this system arises owing to both the choice of the origin and the

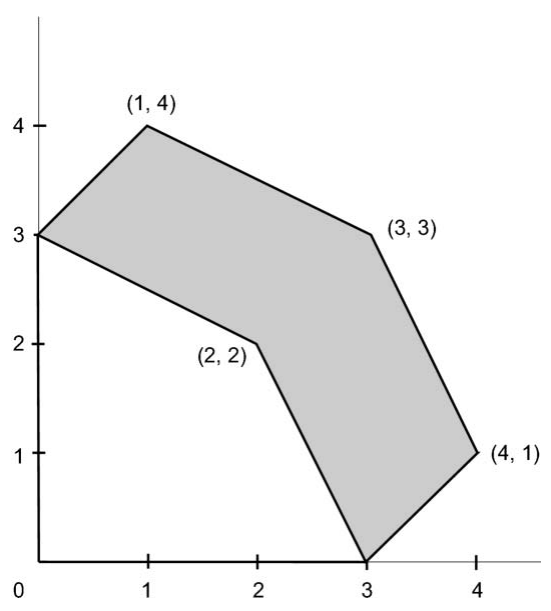

Figure 1

The Newton polytopes for the second equation (the inner polygon) and for the Minkowski sum of the two polytopes (the outer polygon) of system (A5) in the supplementary material for $N=3$. The difference polygon, whose surface area is equal to the mixed volume for the system, is shown in gray. substitution of the coordinates by the elementary symmetric polynomials.

We demonstrated previously that for the origin in the center of mass, solutions of system (A5) can be divided into groups of $2 N$ non-unique structures (Shkel et al., 2011). The $2 N$ structures within each group are obtained from a single structure by $N$ operations of the origin shift and, for each of these $N$ origin locations, by changing the signs of all coordinates to the opposite, i.e. the $2 N$ structures in each group are either congruent or enantiomeric to each other. We define the structures within each of these groups of $2 N$ structures as non-unique; consequently, the number of unique structures equals the number of such groups, i.e. the total number of structures divided by $2 N$. The number of unique structures is given in Table 1 . This number appears to increase exponentially with increasing $N$, indicating that the ambiguity of the structure determination from a minimum of perfectly measured intensities increases exponentially with the structure size. The behavior of this ambiguity for large $N$ is analyzed in $\S 2.4$.

\subsection{Non-homometric ambiguity of structures obtained from a minimum of intensities}

Even in the ideal case when all intensities are available and measured without experimental uncertainty, the structure determination problem does not have a unique solution. It was recognized by Patterson that multiple structures that are described by the same set of interatomic distances (called homometric) can yield the same complete set of intensities. Therefore, in addition to homometric structures, a minimum set of intensities may yield non-unique structures that are not homometric. We showed here (Table 1) and previously by a different method (Shkel et al., 2011) that for $N=2$ and $N=3$, the minimum set of lowest-resolution intensities always yields a unique one-dimensional crystal structure and that nonunique structures arise for $N>3$ (Shkel et al., 2011). Can two non-unique structures obtained for $N=4$ from $I_{1}, I_{2}$ and $I_{3}$ (Table 1) be non-homometric? The answer to this question is yes. For example, two non-homometric four-atom structures: $\{0.125,0.225,0.725,0.925\}$ and $\{0.041,0.300,0.772,0.888\}$ yield the same three lowest-resolution intensities, $I_{1}=2.62$, $I_{2}=2.38$ and $I_{3}=0.38$. The two structures were obtained from these intensities by solving system (A5) by elementary algebraic methods as described in Shkel et al. (2011) and then equation (A6) numerically. By continuity, non-homometric structures can also be obtained from intensities in some vicinity of this intensity point in the three-dimensional space $\left(I_{1}\right.$, $\left.I_{2}, I_{3}\right)$. The non-homometry of structures obtained from a minimum of intensities is likely a general property. Higherresolution intensities are expected to resolve this type of ambiguity. For $N=4$, the $I_{4}$ values for the two structures are identical; therefore, $I_{4}$ cannot resolve the two structures. The two $I_{5}$ values are well separated: $I_{5}=4.00$ and $I_{5}=1.34$ for the first and the second structure, respectively, resolving the ambiguity. 


\subsection{Crystal structure ambiguity analysis for the origin coinciding with one of the atoms}

Defining the origin so that it coincides with one of the atoms yields an elegant generalization of the dependence of the crystal structure ambiguity for large $N$ within the framework of Bernstein's theorem. Without restricting generality, we set $x_{N}=0$, i.e. $\xi_{N}=1$. Then there are $N-1$ remaining unknown coordinates $\xi_{1}, \ldots, \xi_{N-1}$; therefore, the minimal data set consists of $N-1$ low-resolution intensities given by the following system of $N-1$ equations with $N-1$ unknowns:

$$
I_{h}=\left(1+\sum_{j=1}^{N-1} \xi_{j}^{h}\right)\left(1+\sum_{j=1}^{N-1} \xi_{j}^{-h}\right), h=1, \ldots, N-1
$$

or

$$
\left(1+\sum_{j=1}^{N-1} \xi_{j}^{h}\right)\left(1+\sum_{j=1}^{N-1} \xi_{j}^{-h}\right)-I_{h}=0, h=1, \ldots, N-1 .
$$

The left-hand side of each equation of system (4) is a Laurent polynomial. For a given $h$, this polynomial corresponds to an $(N-1)$-dimensional Newton polytope that is formed by $N(N-1)$ vertices: $2(N-1)$ vertices with one coordinate equal to either $h$ or $-h$ and the rest of the coordinates equal to zero and $(N-1)(N-2)$ vertices with one coordinate equal

\section{(a)}
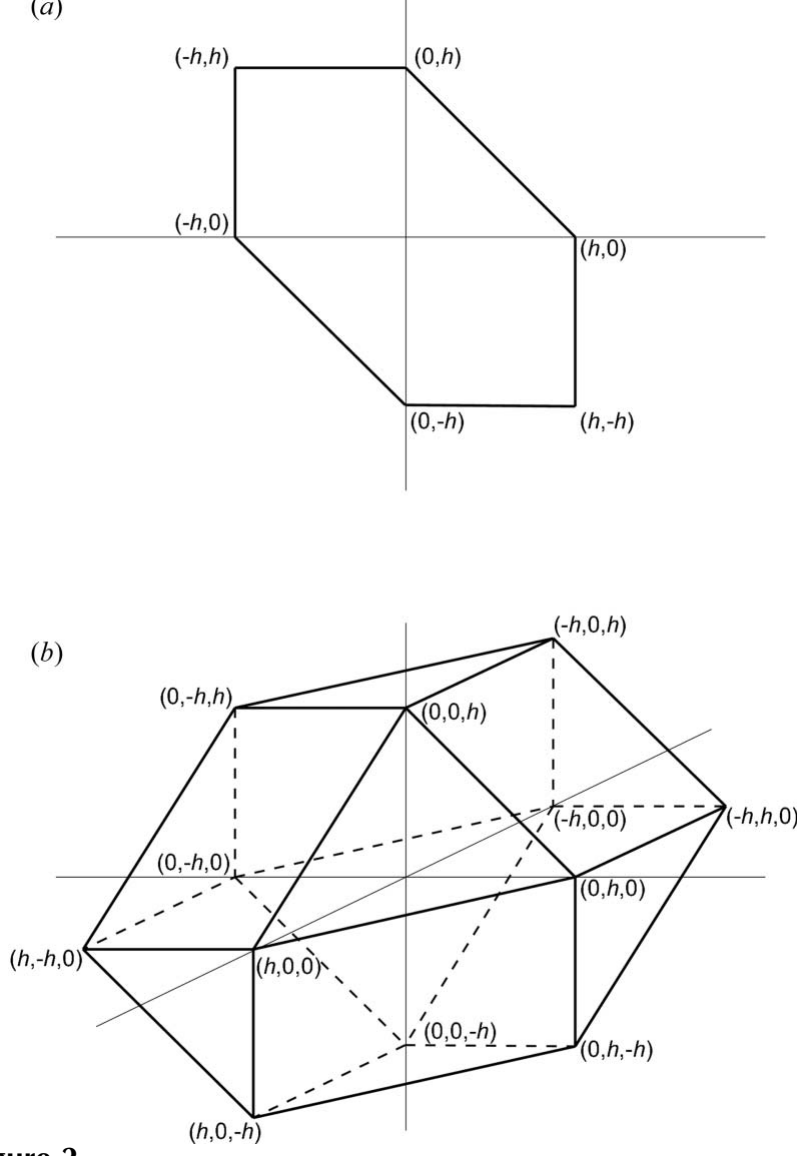

Figure 2

(a) A two-dimensional $h$-polytope. (b) A three-dimensional $h$-polytope. to $h$, another equal to $-h$ and the rest of the coordinates equal to zero (Appendix $C$ in the supplementary material). We will call this Newton polytope an $h$-polytope. The two-dimensional and three-dimensional $h$-polytopes are shown in Fig. 2 . The origin corresponds to the free term in equation (4) and always lies inside these polytopes (Appendix $C$ ); therefore, it does not form a vertex. In Appendix $C$, we prove that $h$-polytopes have a remarkable property: the convex hull of the Minkowski sum of an $h_{1}$-polytope and an $h_{2}$-polytope is an $\left(h_{1}+h_{2}\right)$-polytope. In addition, in Appendix $C$ we prove that the volume of an $M$-dimensional $h$-polytope is

$$
V_{M, h}=h^{M} V_{M, 1}=h^{M} \frac{(2 M) !}{(M !)^{3}},
$$

where $V_{M, 1}$ is the volume of the $M$-dimensional $h$-polytope in which $h=1$. In the calculations for a given number of atoms $N$, one needs to set $M=N-1$ in this expression as $N-1$ is the dimensionality of the problem.

The above properties of an $h$-polytope allow one to apply Bernstein's theorem to system (4) without time-consuming convex hull and $N$-dimensional volume calculations as the mixed volume of this system can be expressed in the closed form. For example, for $N=3$, the mixed volume (area in this case) of system (4) is equal to

$$
V_{\text {mix }}=\left((1+2)^{2}-2^{2}-1^{2}\right) V_{2,1}=4 V_{2,1}=12 .
$$

In the general case of $N$ atoms, the expression in the parentheses in equation (6) is equal to $[(N-1) !]^{2}$. Finally, the mixed volume is

$$
V_{\text {mix }}=[(N-1) !]^{2} V_{N-1,1}=\frac{[2(N-1)] !}{(N-1) !} .
$$

The mixed volume values for $N \leq 12$ are given in Table 1 .

In contrast to system (A5), system (4) contains coordinates of individual atoms explicitly as independent variables. Because the origin can be placed at any one of the $N$ atomic coordinates and the rest of the coordinates can be permuted without changing the functional form of system (4), its number of solutions should be a multiple of $N$ !. Moreover, for each of these $N$ ! structures, an enantiomer whose coordinates have the opposite sign is also a solution of system (4). Therefore, all solutions of this system can be divided into groups of $2 N$ ! nonunique structures. Therefore, the ratio of the mixed volume to $2 N$ ! approximates the number of unique structures:

$$
\frac{V_{\mathrm{mix}}}{2 N !}=\frac{[2(N-1)] !}{2 N !(N-1) !}
$$

These values for $N \leq 12$ are given in Table 1 . One can see from this table that even though for this origin definition the mixed volume calculations do not yield the exact root number for this system, they are a very good approximation. The closed form of equation (8) allows us to apply Stirling's approximation to obtain the behavior of the number of solutions for large $N$ :

$$
\frac{[2(N-1)] !}{2 N !(N-1) !} \stackrel{N>>1}{\simeq} \frac{4^{N}}{8 \pi^{1 / 2} N^{3 / 2}}
$$


Equation (9) demonstrates that the ambiguity of onedimensional structure determination from the minimum of diffraction intensities increases exponentially, in line with observations made in $\S 2.2$ for $N \leq 8$.

\section{Discussion}

Development of direct methods that now allow researchers to determine routinely most crystal structures of small molecules revolutionized the field of crystallography, from their first applications 60 years ago to modern user-friendly software implementations. The field of crystal structure determination was hampered early on by the notion that the correspondence between the structure and the observed intensity data is not one-to-one. Intensities can be generated from a given structure unambiguously, whereas even an idealized complete infinite set of intensities cannot yield a unique structure. This ambiguity is comprised of congruent structures that are related by some symmetry and homometric structures (Patterson, 1939, 1944) that are characterized by the same set of interatomic distances. The number of homometric multiplets increases with increasing structure size (Bullough, 1963). In addition, errors in intensities lead to quasi-homometric structures that produce the same intensities within these errors. The success of direct methods overcame this initial psychological barrier (Hauptman, 1986). Nevertheless, direct methods are intrinsically probabilistic and are limited to crystals of small molecules or, at best, to very small proteins for which data of exceptional quality are available. Because of their probabilistic nature, these methods do not always yield a structure even for small molecules (Langs \& Hauptman, 2011). Therefore it would be highly desirable to develop an exhaustive deterministic approach that would guarantee obtaining the structure of interest from good-quality data without a limitation on the size of the structure. This idea recently prompted us to revisit some poorly understood basic crystallographic notions, by methods of elementary and modern computational algebra.

In this study we ask arguably the most basic question: how many one-dimensional structures can be obtained from a minimum of perfectly determined intensities of lowest resolutions? The answer is somewhat unexpected: exponentially many. This, of course, means that the minimum set of intensities ( $N-1$ intensities for a one-dimensional crystal) is fundamentally insufficient for structure determination even when the intensities are error free. However, the success of probabilistic direct methods indicates that adding higherresolution intensities to the input data set is expected to resolve this ambiguity very efficiently. In fact, overdetermining the problem is essential for successful application of the direct methods (Hauptman \& Karle, 1957; Karle \& Hauptman, 1957). The dependence of the structure ambiguity on the number of intensities beyond the minimum is being currently investigated by our group. Karle and Hauptman illustrated how one intensity beyond the minimum set resolved the ambiguity for a one-dimensional three-atom structure of nonequal atoms (Hauptman \& Karle, 1951). For equal atoms, we previously demonstrated that the ambiguity exists for $N \geq 4$ (Shkel et al., 2011) and in this study showed how it gets resolved by an additional intensity. We propose that the efficient resolution of error-free intensities to a unique structure can be achieved by a sufficiently large number of intensities owing to the fact that the ambiguity is likely non-homometric. This proposition is consistent with the previous notion that a finite set of error-free intensities that obey certain determinant conditions is sufficient for structure determination (Cervellino \& Ciccariello, 1996). Homometry imposes a very special set of constraints and relationships on the interatomic distances, as exemplified by homometric structures generated from cyclotomic sets (Patterson, 1944), whereas a random structure is unlikely to satisfy these relationships. Another serious issue noted previously is that the errors in intensities are a source of great structure ambiguity if the number of intensities is sufficiently small (in line with our results); whereas when the number of the inaccurate intensities is too large, the polynomial equations become inconsistent and their system cannot yield a solution (Cervellino \& Ciccariello, 1996). Ongoing research in our group using modern numerical algebra is aimed at overcoming these obstacles.

In summary, we developed a novel analysis of ambiguity of one-dimensional crystal structures of equal point atoms, by application of methods of modern computational algebraic geometry to the century-old problem of crystal structure determination solely from algebraic intensities. We introduced a geometrical object, an $h$-polytope, which due to its remarkable properties demonstrated here yielded a generalization of this analysis to any number of atoms.

The authors thank Dr Karl Fischer for stimulating discussions and critical reading of the manuscript and the University of Michigan College of Pharmacy for financial support.

\section{References}

Avrami, M. (1938). Phys. Rev. 54, 300-303.

Barber, C. B., Dobkin, D. P. \& Huhdanpaa, H. (1996). ACM T. Math. Software, 22, 469-483.

Bullough, R. K. (1963). Nature (London), 200, 250.

Cervellino, A. \& Ciccariello, S. (1996). Riv. Nuovo Cimento, 19, 1-59. Cervellino, A. \& Ciccariello, S. (1999). Z. Kristallogr. 214, 739-750.

Cervellino, A. \& Ciccariello, S. (2001). J. Phys. A Math. Gen. 34, 731755.

Cervellino, A. \& Ciccariello, S. (2005). Acta Cryst. A61, 494-500.

Fischer, K. F. \& Pilz, K. (1997). Acta Cryst. A53, 475-483.

Gillis, J. (1948a). Acta Cryst. 1, 76-80.

Gillis, J. (1948b). Acta Cryst. 1, 174-179.

Harker, D. \& Kasper, J. S. (1947). J. Chem. Phys. 15, 882-884.

Harker, D. \& Kasper, J. S. (1948). Acta Cryst. 1, 70-75.

Hauptman, H. (1986). Angew. Chem. Int. Ed. Engl. 25, 603-613.

Hauptman, H. \& Karle, J. (1951). Acta Cryst. 4, 383.

Hauptman, H. \& Karle, J. (1957). Acta Cryst. 10, 267-270.

Karle, J. \& Hauptman, H. (1957). Acta Cryst. 10, 515-524.

Langs, D. A. \& Hauptman, H. A. (2011). Acta Cryst. A67, 430-434.

Miller, R., Gallo, S. M., Khalak, H. G. \& Weeks, C. M. (1994). J. Appl.

Cryst. 27, 613-621.

Ott, H. (1927). Z. Kristallogr. 66, 136-153. 


\section{research papers}

Patterson, A. L. (1939). Nature (London), 143, 939-940.

Patterson, A. L. (1944). Phys. Rev. 65, 195-201.

Pauling, L. \& Shappell, M. D. (1930). Z. Kristallogr. 75, 128-142.

Pilz, K. \& Fischer, K. F. (1998). Acta Cryst. A54, 273-282.

Pilz, K. \& Fischer, K. F. (1999). Acta Cryst. A55, 564.

Pilz, K. \& Fischer, K. F. (2000). Z. Kristallogr. 215, 640-649.
Rappleye, J., Innus, M., Weeks, C. M. \& Miller, R. (2002). J. Appl. Cryst. 35, 374-376.

Sheldrick, G. M. (2008). Acta Cryst. A64, 112-122.

Shkel, I. A., Lee, H. S. \& Tsodikov, O. V. (2011). Acta Cryst. A67, $292-$ 296.

Weeks, C. M. \& Miller, R. (1999). J. Appl. Cryst. 32, 120-124. 